\title{
Experimental Validation of Analytical Model for Lamb Wave Interaction with Geometric Discontinuity
}

\author{
Banibrata Poddar ${ }^{\text {a) }}$ and Victor Giurgiutiu ${ }^{\text {a) }}$ \\ ${ }^{a}$ Department of Mechanical Engineering, University of South Carolina, USA
}

\begin{abstract}
Non-destructive testing methods based on ultrasonic waves are one of the most popular methods for damage detection in structures. Of these ultrasonic waves Lamb waves are of particular interest for the inspection of large structures for various reasons. Therefore scattering of Lamb waves from flaws has generated a considerable amount of research over last couple of decades. Most of the work has been done using computational tools like Finite Element Methods and experimental technique. In this paper an analytical approach is presented to develop a fundamental understanding of the scattering of Lamb waves from geometric discontinuities in 2 dimensions. We have considered simplest of all geometric discontinuity - a step, as this fundamental understanding can easily be extended to corrosion or crack.
\end{abstract}

Keywords: structural health monitoring, nondestructive evaluation, guided waves, Lamb waves, analytical model, damage detection, piezoelectric wafer active sensor (PWAS)

\section{INTRODUCTION}

The goal of a structural health monitoring (SHM) system is to implement processes to detect and characterize damages in engineering structures. To develop a SHM system based on Lamb wave propagation we first need to understand how Lamb waves interact with different damage types. In this study we attempted to develop an understanding of how Lamb waves interact with geometric discontinuities using analytical modelling technique. For simplicity we considered only a 2D step type geometric discontinuity as this addresses the fundamental challenge of satisfying the continuous boundary conditions at the location of the discontinuity without getting into a much complicated geometric condition. Also this case can easily be extended to the case of real life damages such as crack or a delamination.

Numerous studies have been done to solve this problem [1-8]. In most of these studies finite element based approach compared with experimental results were used to understand the scattered field. In other studies [3] modal decomposition method was used to predict the scattered wave field. But the continuous boundary condition was scaled down to discrete points across the boundary to satisfy the boundary conditions as it is very challenging to satisfy the continuous boundary condition. In 1982, Gregory et-al $[9,10]$ introduced a method called 'projection method' to satisfy the continuous boundary conditions. This method was also capable of predicting the singularity in stresses in case of a geometric discontinuity [4]. A modified form of projection method was used by Grahn in 2003 [6]. Although this scalar projection method was simpler and able to predict the scattered wave field, the convergence was slow [7]. In 2012 Moreau et-al [7,11] used a vector projection method which had faster convergence and showed promising results.

In this study a form of modal expansion of Lamb modes is used to calculate the scattered wave field. To satisfy the continuous boundary conditions we have used vector project of the boundary conditions to simplify them. Since Rayleigh-Lamb equation has infinite number of complex roots resulting in infinite number of complex Lamb modes, for the sake of convenience we will call this method complex mode expansion with vector projection (CMEP). For verification of CMEP we used FEM. We created an FEM model to obtain scattered wave field from the step and compared it with CMEP. We have also presented our experimental procedure for verification of the results obtained from CMEP. 


\section{SOLVING RAYLEIGH-LAMB EQUATION}

For CMEP we need to obtain all possible roots of Rayleigh-Lamb equation including infinite number of complex roots. Since Rayleigh-Lamb equation is a transcendental equation and does not have closed form solution, we have found the roots of this equation over large domain of complex plane by using recursive iterative algorithms written in MATLAB. The algorithm can converge to root within specified accuracy with certainty. This is important as we need to use correct root of this equation for CMEP.

$$
\frac{\tan \eta_{P} d}{\tan \eta_{S} d}=-\left[\frac{\left(\xi^{2}-\eta_{S}^{2}\right)^{2}}{4 \xi^{2} \eta_{P} \eta_{S}}\right]^{ \pm 1} \quad \eta_{P}^{2}=\frac{\omega^{2}}{c_{P}^{2}}-\xi^{2}, \eta_{S}^{2}=\frac{\omega^{2}}{c_{S}^{2}}-\xi^{2}
$$

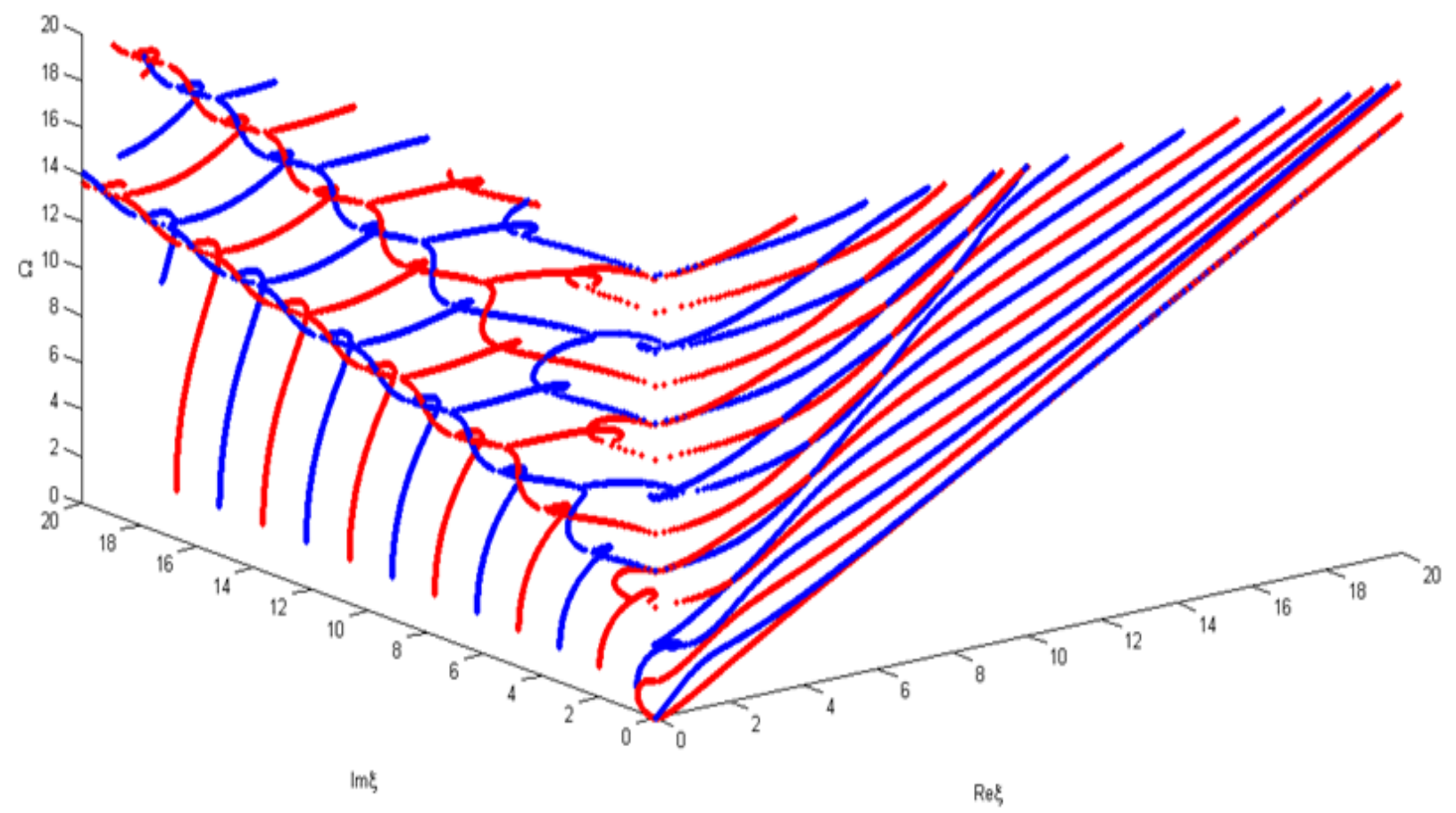

FIGURE 1. Roots of Rayleigh-Lamb equation. Symmetric modes are plotted in blue and antisymmetric modes are plotted in red. 


\section{INTERACTION OF LAMB WAVES WITH STEP}

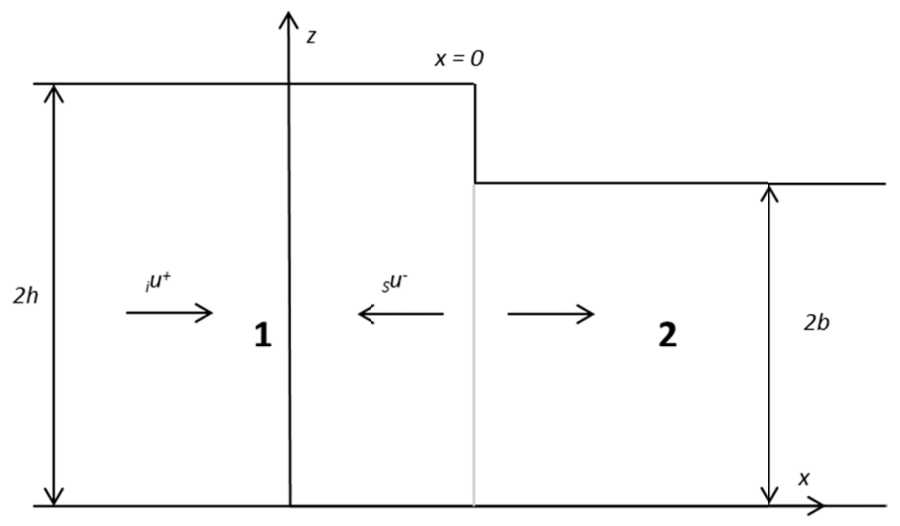

FIGURE 2. Illustration of the step and interacting Lamb waves.

For Lamb waves we consider the incident wave field as,

and the scattered field as,

$$
\begin{aligned}
& \overrightarrow{u_{i}}=\sum_{j=1}^{\infty} C_{j 1}^{+} \overrightarrow{u_{j}} e^{i\left(+\xi_{j} x-\omega t\right)} \\
& \overrightarrow{\sigma_{i}}=\sum_{j=1}^{\infty}{ }_{i} C_{j 1}^{+} \overrightarrow{\sigma_{j}} e^{i\left(+\xi_{j} x-\omega t\right)}
\end{aligned}
$$

$$
\begin{aligned}
& \overrightarrow{u_{s}}=\sum_{j=1}^{\infty}\left({ }_{s} C_{j}^{+} \overrightarrow{{ }_{2}} \overrightarrow{ }_{j} e^{i\left(+\xi_{j} x-\omega t\right)}+{ }_{s} C_{j 1}^{-} \overrightarrow{u_{j}} e^{i\left(-\xi_{j} x-\omega t\right)}\right) \\
& \overrightarrow{\sigma_{s}}=\sum_{j=1}^{\infty}\left({ }_{s} C_{j}^{+} \overrightarrow{{ }_{2} \sigma_{j}} e^{i\left(+\xi_{j} x-\omega t\right)}+{ }_{s} C_{j}^{-} \overrightarrow{\sigma_{j}} e^{i\left(-\xi_{j} x-\omega t\right)}\right)
\end{aligned}
$$

where, subscript " $i$ " stands for incident waves and " $s$ " stands for scattered waves with superscript "+" for waves travelling in $+x$ direction and "-" for waves travelling in $-x$ direction. Also the subscript " $j$ " represents different wave modes.

The boundary conditions applied at the step are displacement boundary condition,

$$
\vec{u}=\vec{u}, \quad 0<z<2 b, \quad x=0
$$

and traction boundary conditions,

$$
\begin{aligned}
& \overrightarrow{{ }_{1} t}=\left\{\begin{array}{ccc}
0, & 2 b<z<2 h, & x=0 \\
\overrightarrow{{ }_{2} t}, & 0<z<2 b, & x=0
\end{array}\right. \\
& \Rightarrow \vec{\sigma}=\left\{\begin{array}{ccc}
0, & 2 b<z<2 h, & x=0 \\
\overrightarrow{{ }_{2} \sigma}, & 0<z<2 b, & x=0
\end{array}\right.
\end{aligned}
$$

We project them onto an appropriate complete orthogonal vector space to remove the $\mathrm{z}$ dependence of the boundary condition. To take advantage of the orthogonality of stress and displacement mode shapes of Lamb wave modes, we choose ${ }_{1} \bar{u}_{j}=\operatorname{conj}\left(\overrightarrow{ }_{1} \vec{u}_{j}\right)$ as the projection vectors for the stress boundary conditions and ${ }_{2} \bar{\sigma}_{j}=\operatorname{conj}\left(\overrightarrow{{ }_{2} \sigma_{j}}\right)$ as the projection vectors for the displacement boundary conditions. The final forms of the boundary conditions independent of $\mathrm{z}$ are, 


$$
\begin{aligned}
& \int_{0}^{2 b}\left(\overrightarrow{{ }_{1} u}-\overrightarrow{{ }_{2} u}\right)_{2} \bar{\sigma}_{j} d z=0 \Rightarrow\left\langle\left(\overrightarrow{{ }_{2} u_{S}}-\overrightarrow{{ }_{1} u_{S}}\right),{ }_{2} \bar{\sigma}_{j}\right\rangle=\left\langle\overrightarrow{ }_{1}{ }_{i},{ }_{2} \bar{\sigma}_{j}\right\rangle ; \quad x=0 \\
& \int_{0}^{2 h} \overrightarrow{ }_{1}{ }_{1} \bar{u}_{j} d z=\int_{0}^{2 b} \overrightarrow{ }_{2}{ }_{1} \bar{u}_{j} d z \Rightarrow\left\langle\left(\overrightarrow{{ }_{2} \sigma_{S}}-\overrightarrow{{ }_{1} \sigma_{S}}\right),{ }_{1} \bar{u}_{j}\right\rangle=\left\langle\overrightarrow{{ }_{1}},{ }_{1} \bar{u}_{j}\right\rangle ; \quad x=0
\end{aligned}
$$

This leads us to the set of linear equations expressed as,

$$
[A]_{2 j \times 2 j}\{C\}_{2 j \times 1}=\{B\}_{2 j \times 1}
$$

For numerical results we need to determine the maximum number of roots of Rayleigh-Lamb equation to be considered which should give us reasonably accurate result. Figure 3 shows the convergence of the modal participation factors of the first three modes of the Lamb waves and we can see that 27 modes are more than enough for the result to converge. Then the above equation can be easily solved using matrix inversion in MATLAB.

\section{Convergence of the reflection and transmission cefficients}

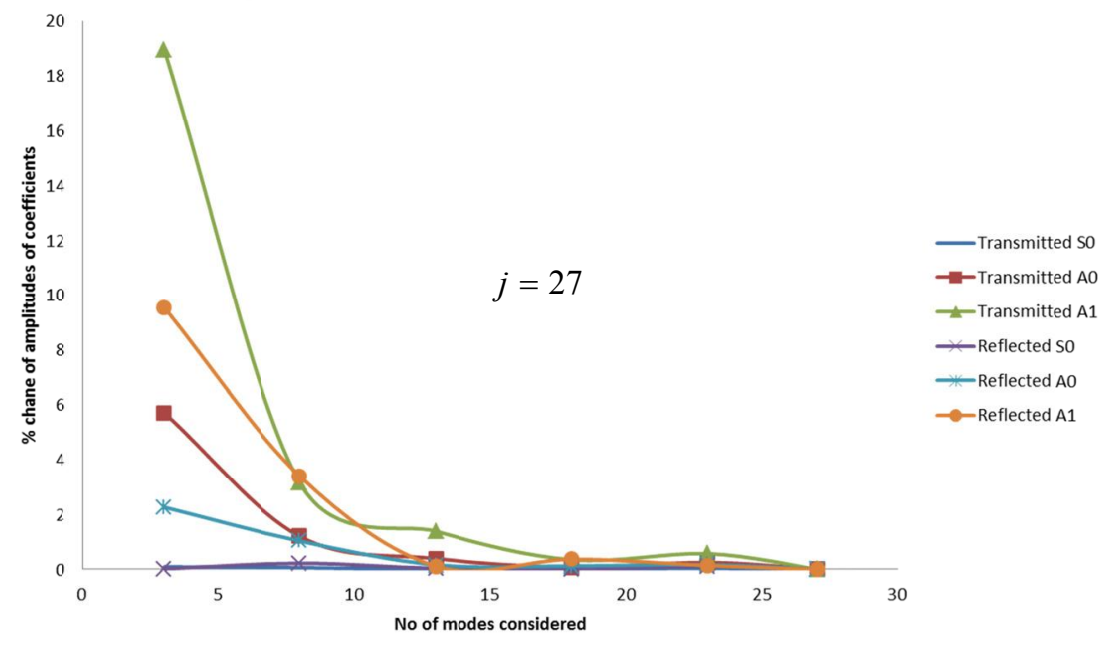

FIGURE 3. Convergence study.

\section{FEM MODEL}

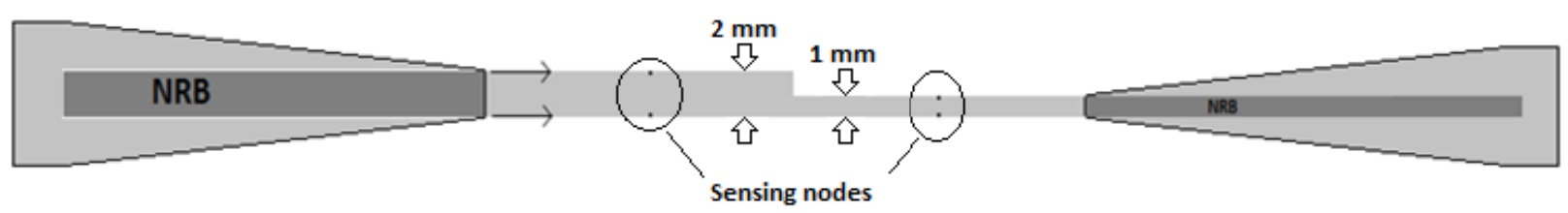

FIGURE 4. A FEM model.

To model interaction of Lamb waves with step we used 2D plane strain model. To obtain the scatter coefficients as a function of frequency we did harmonic analysis. Harmonic analysis normally will produce standing wave field. To get a transient response we introduced non reflective boundary at the edges of the model such that they do not reflect any wave. Therefore doing a harmonic analysis with nonreflecting boundary enabled us to obtain the scatter coefficients as a function of time. The nonreflecting boundary was created using linear spring damper element attached to the surface of the boundaries. The damping constants were increased gradually such that no reflection occurs at the nonreflecting boundary. The simulation was done for incident $\mathrm{S}_{0}$ Lamb wave mode.

From Fig. 5 we can see that the results from FEM and CMEP match perfectly. The minor noise in the FEM data was due to the numerical noise generated by round-off errors. 


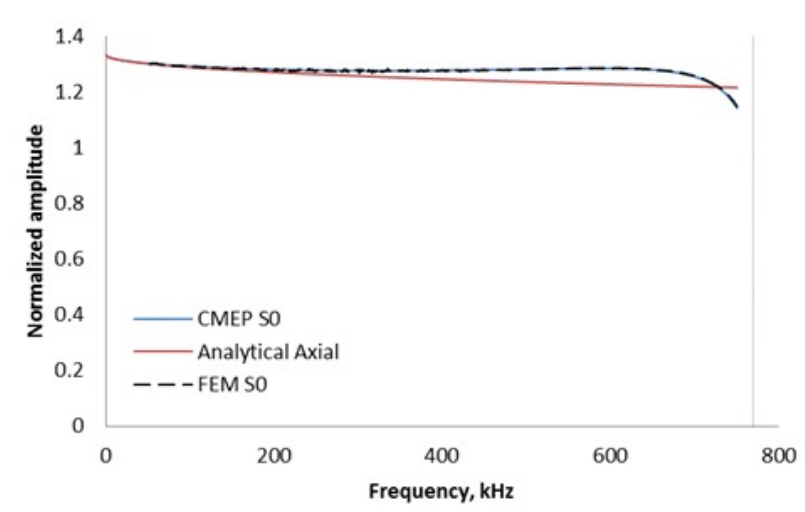

(a)

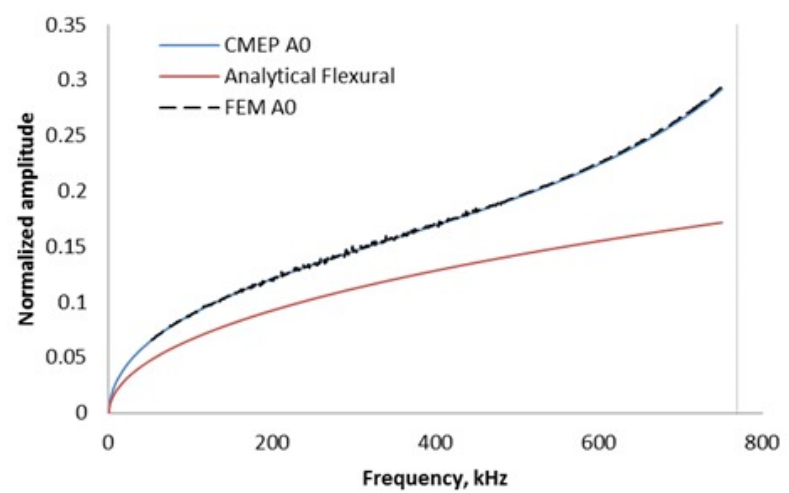

(c)

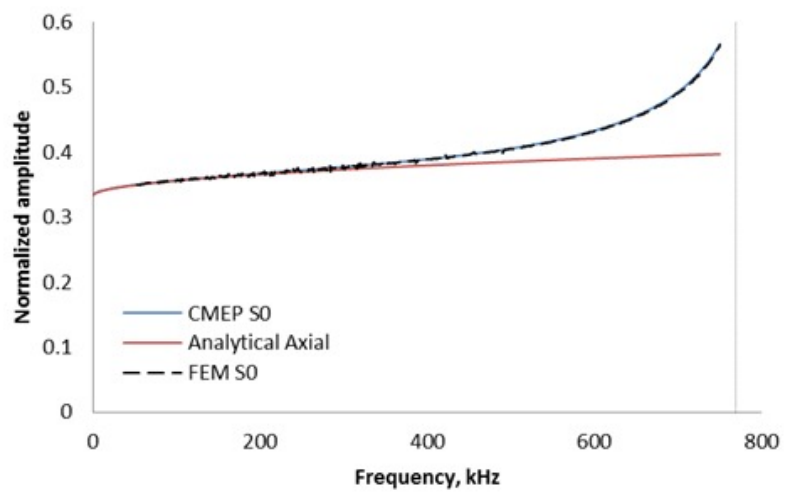

(b)

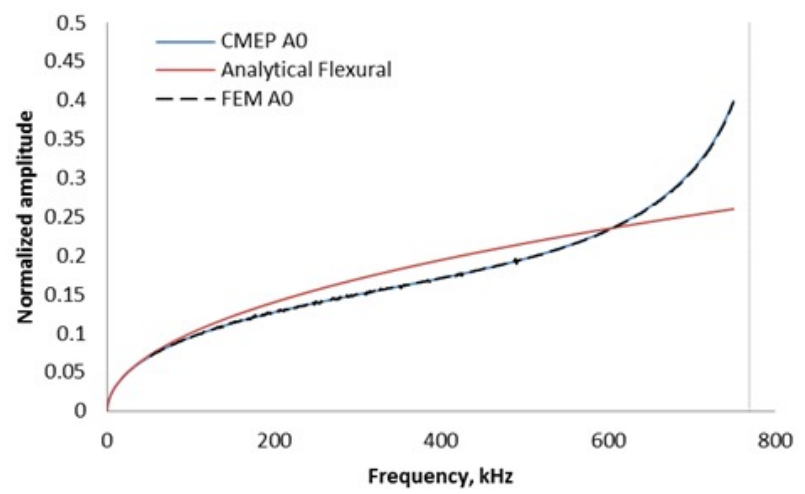

(d)

FIGURE 5. Comparison of top surface $x$ displacement (a), (c) transmitted waves and (b), (d) reflected waves.

\section{PROCEDURE FOR EXPERIMENTAL VERIFICATION}

PWAS

PWAS

FIGURE 6. Setup for experiment

For experimental verification we chose to use piezoelectric wafer active sensors (PWAS) to actuate Lamb waves in an aluminum plate because of ease of use of PWAS and its reliability. To excite symmetric or antisymmetric mode selectively, we used two PWAS bonded to the plate; one on the top surface and one on the bottom surface. This will enable us to excite both of them in phase or out of phase to excite symmetric and antisymmetric Lamb waves mode. One of the many challenges of this experiment is to generate a straight crested wave front using finite dimensional actuators. We used $5 \mathrm{~mm} \times 40 \mathrm{~mm}$ PWAS to excite an almost straight crested wave front (Figure 6).

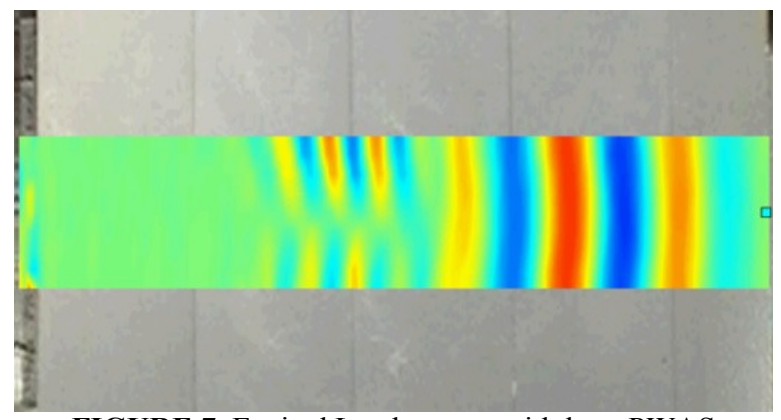

FIGURE 7. Excited Lamb waves with long PWAS 
The next challenge was to eliminate the edge reflections to be able to detect the reflected and transmitted waves from the step. To achieve this we tried to recreate the nonreflecting boundary similar to the FEM model in real life experiment. We used soft modeling clay to create an absorbing boundary to absorb all edge reflections. The thickness of the clay was increased gradually similar to the damping coefficient in NRB in the FEM model such that there is no reflection from the clay-aluminum boundary (Figure 8).

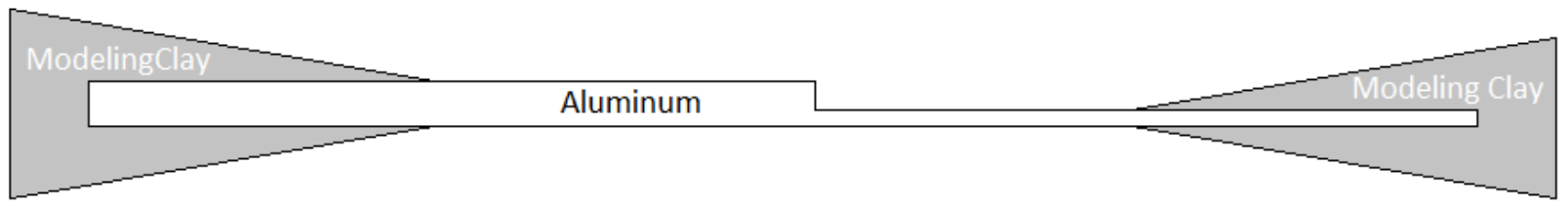

FIGURE 8. Absorbing boundary to eliminate reflections

With the absorbing boundary around the plate the final setup looks like Figure 9.

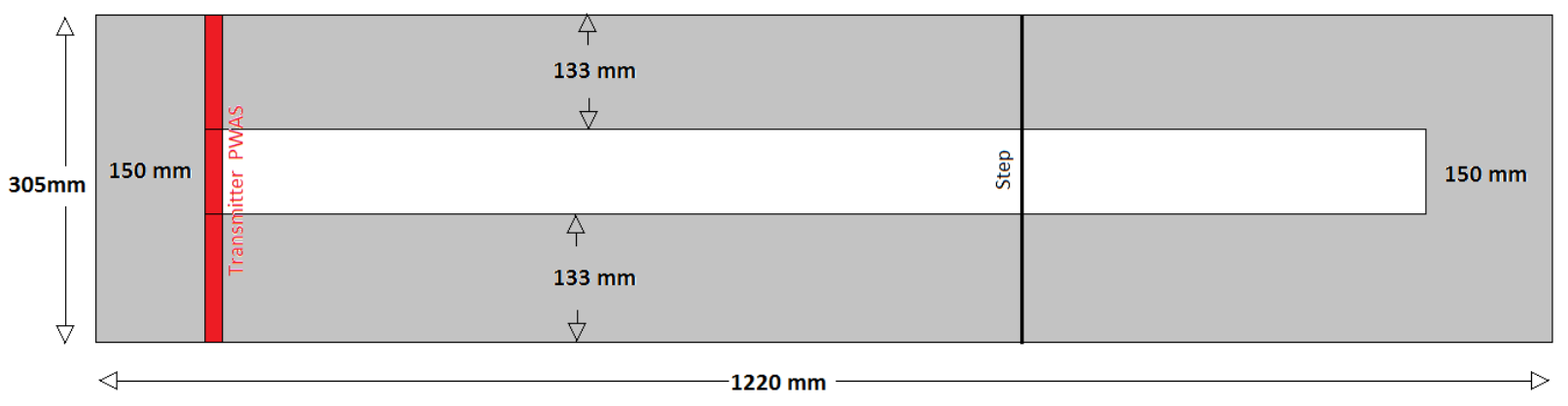

FIGURE 9. Schematics of the final setup

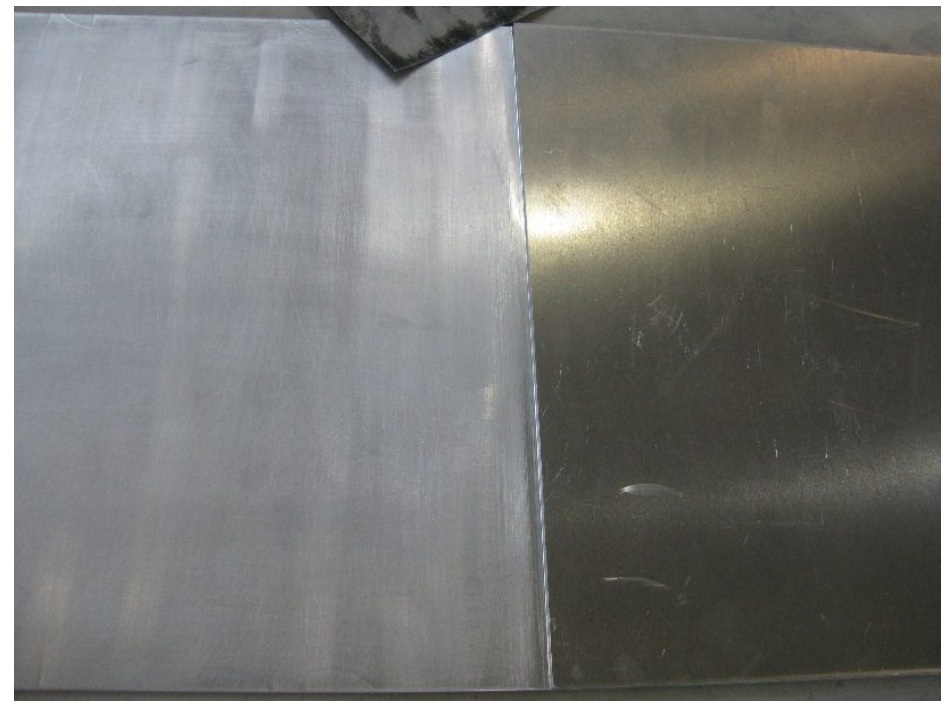

FIGURE 10. Stepped plate specimen

We chose Al6061 for the material for the plate as it has good machinability. We needed relatively thick plate so that we can machine it to create a step in half of the plate (Figure 10). We used a $4.86 \mathrm{~mm}$ thick plate to create the step by milling it. After applying the modeling clay the finished specimen looks like Figure 11. 


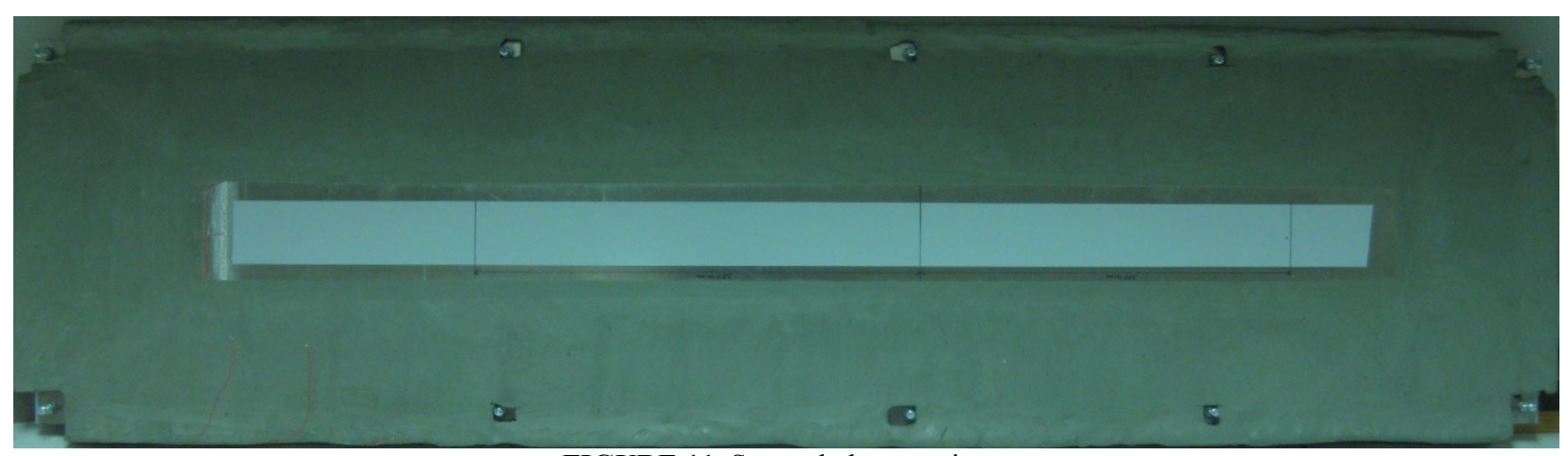

FIGURE 11. Stepped plate specimen

We used Laser Doppler Vibrometer to measure the out of plane velocity of the wave field. From figure 12 we can see that after application of the clay boundary and tuning the top-bottom PWAS, we have created a straight crested A0 mode.

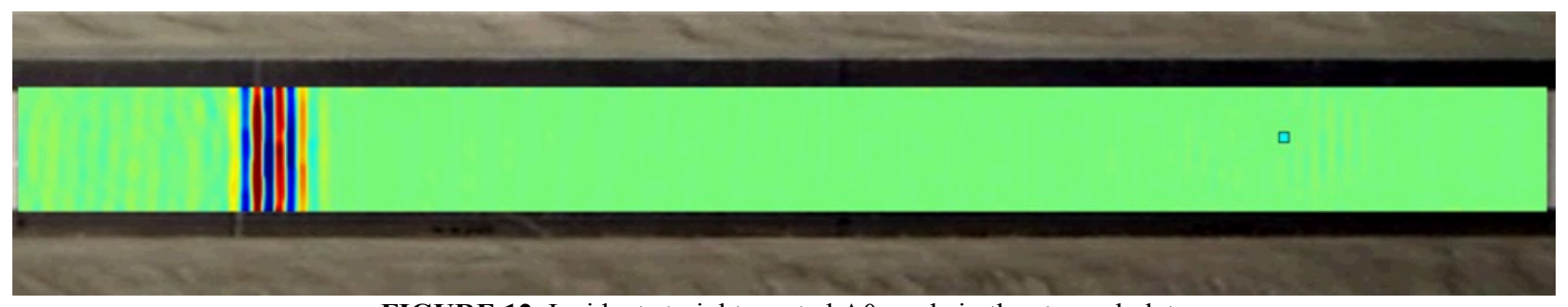

FIGURE 12. Incident straight crested A0 mode in the stepped plate

\section{SUMMARY AND CONCLUSION}

A robust algorithm was developed to find roots of Rayleigh-Lamb equation over a very complex domain with controlled accuracy. An analytical, CMEP was developed to predict the scatter field of Lamb wave produced by a geometric discontinuity. An FEM model was made to compare and validate CMEP. The numerical model proved CMEP to be an accurate prediction of the scatter field. Vice versa CMEP confirmed the validity of the result of FEM simulation. This also proves that CMEP can be used to check for FEM model validity. CMEP also shows that it is possible to obtain the scatter field of Lamb wave analytically. CMEP can also predict the local field of vibration in terms of nonpropagating Lamb wave modes. An experimental procedure was developed selective mode tuning of S0 and A0 modes of Labs waves along with straight crested wave front. The interaction of the wave with a step was then recorded using Laser Doppler Vibrometer.

The technique of vector project can be extanded for 3D geometric discontinuities and real damages such as cracks and delamination. Therefore CMEP can be extended to model scattering of Lamb waves in 3D geometries without much difficulties.

\section{ACKNOWLEDGMENTS}

Support from office of Naval Research \# N00014-11-1-0271, Dr. Ignacio Perez, Technical Representative and Air Force Office of Scientific Research \#FA9550-11-1-0133, Dr. David Stargel, Program Manager; are thankfully acknowledged. 


\section{REFERENCES}

1. D. N. Alleyne and P. Cawley, "The interaction of Lamb waves with defects," IEEE Trans Ultrason Ferroelectr Freq Control, 39 (3), 381-97 (1992).

2. D. N. Alleyne and P. Cawley, "The effect of discontinuities on the long-range propagation of Lamb waves in pipes," Proc Inst Mech Eng, Part E J Process Mech Eng., 210-217 (1996).

3. M. Castaings, E. Le Clezio, and B. Hosten, "Modal decomposition method for modeling the interaction of Lamb waves with cracks," J Acoust Soc Am., 112 (6), 2567-2582 (2002).

4. M. A. Flores-López and Gregory R. Douglas, "Scattering of Rayleigh-Lamb waves by a surface breaking crack in an elastic plate," J Acoust Soc Am., 119 (4), 2041 (2006).

5. E. V. Glushkov, N. V. Glushkova, and O. N. Lapina, "Diffraction of Normal Modes in Composite and Stepped Elastic Waveguides. J Appl Math Mech., 62 (2), 275-280 (1998).

6. T. Grahn, "Lamb wave scattering from a circular partly through-thickness hole in a plate,". Wave Motion. 37 (1), 63-80 (2003).

7. L. Moreau, M. Caleap, A. Velichko, and P. D. Wilcox, "Scattering of guided waves by flat-bottomed cavities with irregular shapes," Wave Motion, 49 (2), 375-387 (2012).

8. S. Rokhlin, "Diffraction of Lamb waves by a finite crack in an elastic layer," J Acoust Soc Am., 67 (4), 1157-1165 (1980).

9. $\quad$ Gregory R. Douglas and I. Gladwell, "The cantilever beam under tension, bending or flexure at infinity," $J$ Elast. 12 (4), 317-343 (1982).

10. Gregory R. Douglas and I. Gladwell, "The reflection of a symmetric Rayleigh-Lamb wave at the fixed or free edge of a plate," J Elast., 13, 185-206 (1983).

11. L. Moreau, M. Caleap, A. Velichko, and P. D. Wilcox, "Scattering of guided waves by through-thickness cavities with irregular shapes," Wave Motion, 48 (7), 586-602 (2011). 